\title{
Editorial
}

\section{Filtering and Control for Unreliable Communication: The Discrete-Time Case}

\author{
Guoliang Wei, ${ }^{1}$ Lifeng $\mathrm{Ma}^{2}$ and Zidong Wang ${ }^{3,4}$ \\ ${ }^{1}$ Shanghai Key Lab of Modern Optical System, School of Optical-Electrical and Computer Engineering, \\ University of Shanghai for Science and Technology, No. 516, Jungong Road, Shanghai 200093, China \\ ${ }^{2}$ School of Automation, Nanjing University of Science and Technology, Nanjing, Jiangsu 210094, China \\ ${ }^{3}$ Department of Computer Science, Brunel University, Uxbridge, Middlesex UB8 3PH, UK \\ ${ }^{4}$ NAAM Research Group, Department of Mathematics, King Abdulaziz University, Jeddah 21589, Saudi Arabia
}

Correspondence should be addressed to Guoliang Wei; guoliang.wei1973@gmail.com

Received 8 April 2014; Accepted 8 April 2014; Published 30 April 2014

Copyright (C) 2014 Guoliang Wei et al. This is an open access article distributed under the Creative Commons Attribution License, which permits unrestricted use, distribution, and reproduction in any medium, provided the original work is properly cited.

In the past decades, communication networks have been extensively employed in many practical control systems, such as manufacturing plants, aircraft, and spacecraft to transmit information and control signals between the system components. When a control loop is closed via a serial communication channel, a networked control system (NCS) is formed. NCSs have become very popular for their great advantages over traditional systems (e.g., low cost, reduced weight, and power requirements, etc.). Generally, it has been implicitly assumed that the communication between the system components is perfect; that is, the signals transmitted from the plant always arrive at the filter or controller without any information loss. Unfortunately, such an assumption is not always true. For example, a common feature of the NCSs is the presence of significant network-induced delays and data losses across the networks. Therefore, an emerging research topic that has recently drawn much attention is how to cope with the effect of network-induced phenomena due to the unreliability of the network communication. This special issue aims at bringing together the latest approaches to understand, filter, and control for discrete-time systems under unreliable communication. Potential topics include but are not limited to (a) multiobjective filtering or control, (b) network-induced phenomena, (c) stability analysis, (d) robustness and fragility, and (e) applications in real-world discrete-time systems.

We have solicited submissions to this special issue from control engineers, electrical engineers, computer scientists, and mathematicians. After a rigorous peer review process,
13 papers have been selected that provide overviews, solutions, or early promises to manage, analyze, and interpret dynamical behaviours of discrete-time systems. These papers have covered both the theoretical and practical aspects of discrete-time systems in the broad areas of dynamical systems, mathematics, statistics, computer science, and practical engineering.

This special issue starts with a survey paper on the recent advances of control and filtering for parametervarying systems via probability-dependent method. Specifically, in the paper entitled "A survey on gain-scheduled control and filtering for parameter-varying systems" by G. Wei et al., the focus is to provide a timely review on the recent advances developments on the gain-scheduled control and filtering problems for the parameter-varying systems. First of all, several important algorithms suitable for gainscheduling method are recalled including gain-scheduled proportional-integral-derivative (PID) control, $H_{2}, H_{\infty}$, and mixed $H_{2} / H_{\infty}$ gain-scheduling methods as well as fuzzy gain-scheduling techniques. Then, various important parameter-varying system models are reviewed, for which gainscheduled control and filtering issues are usually dealt with. In particular, in view of the randomly occurring phenomena with time-varying probability distributions, some results of recent work based on the probability-dependent gainscheduling methods are also reviewed. Subsequently, some latest results in this area are summarized. Finally, conclusions are drawn and several potential future research directions are outlined. 
During the past decades, the problems of performance analysis of discrete-time systems have received considerable research interests. In the paper entitled "Fairness analysis for multiparty nonrepudiation protocols based on improved strand space" by L. Li et al., the problem of fairness analysis for multiparty nonrepudiation protocols is studied and a new formal analysis method based on improved strand space is firstly presented. Based on the strand space theory, the assumption of free encryption is extended in the new method. The formal definition of fairness in multiparty nonrepudiation protocols is given and the guideline to verify it based on improved strand space is presented. Finally, the fairness of multiparty nonrepudiation protocols is verified with an example of Kremer-Markowitz protocol. The global exponential stability analysis of discrete-time neural networks is discussed in "Global exponential stability of discrete-time neural networks with time-varying delays" by S. Udpin and P. Niamsup, and some global stability criteria of discrete-time neural networks with time-varying delays are proposed. New global stability condition for nonlinear difference equation is derived based on discrete type inequality. The nonlinear discrete systems considered are subject to time-varying delays and independent of delay time.

The design of various controllers has long been the main stream of research topics and much effort has been made for discrete-time systems. In the paper entitled "Adaptive state-feedback stabilization for stochastic nonholonomic mobile robots with unknown parameters" by W. Feng et al., the stabilizing problem of stochastic nonholonomic mobile robots with uncertain parameters is addressed and nonholonomic mobile robots with kinematic unknown parameters are extended to the stochastic case. Based on backstepping technique, adaptive state-feedback stabilizing controllers are designed. A switching control strategy for the original system is also presented. The proposed controllers guarantee the states of closed-loop system are asymptotically stabilized at the zero equilibrium point in probability. In the work entitled "Global output control for a class of inherently higherorder nonlinear time-delay systems based on homogeneous domination approach" by using the homogeneous domination approach, a homogeneous output feedback controller with an adjustable scaling gain is constructed. With the aid of a homogeneous Lyapunov-Krasovskii functional, scaling gain is adjusted to dominate the time-delay nonlinearities bounded by homogeneous growth conditions and render the closed-loop system globally asymptotically stable. In the paper entitled "Event-based control for average consensus of wireless sensor networks with stochastic communication noises" by C. Ji et al., average consensus problem for the wireless sensor networks (WSNs) with fixed and Markovian switching is investigated. Event-based protocol is applied to each sensor node to reach the consensus. An event triggering strategy is designed based on a Lyapunov function. Under the event trigger condition, some sufficient conditions for average consensus in mean square are obtained. Then some numerical simulations are given to illustrate the effectiveness of the results derived.

The past decades have witnessed a flurry of filter design and state estimation problem, which have found successful applications in a wide range of practical engineering domains. In the paper entitled " $H_{\infty}$ filtering of time-varying nonlinear systems with randomly occurring output degradation" by Z. Jia and Y. Sun, the $H_{\infty}$ filtering problem for nonlinear systems with randomly occurring output degradation phenomenon has been dealt. Such a phenomenon is described by a stochastic variable which obeys the Bernoulli distribution with probability known a prior. A sufficient condition is derived for the nonlinear system to reach the required $H_{\infty}$ performance. An iterative algorithm is then proposed to obtain the filter parameters recursively by solving the corresponding linear matrix inequality. An adaptive Kalman estimator is designed in "Adaptive Kalman estimation in target tracking mixed with random one-step delays, stochastic-bias measurements, and missing measurements" by S. Chen et al., whose objective is concerned with the estimation problem for linear discretetime stochastic systems with mixed uncertainties involving random one-step sensor delay, stochastic-bias measurements, and missing measurements. Three Bernoulli distributed random variables are employed to described the uncertainties. All the three uncertainties in the measurement have certain probability of occurrence in the target tracking system. And then, an adaptive Kalman estimation is proposed to deal with this problem. The adaptive filter gains can be obtained in terms of solutions to a set of recursive discretetime Riccati equations. The work entitled "Experimental and analytical studies on improved feedforward ML estimation based on LS-SVR" by X. Liu and H. Yu is concerned with the estimation problem. Maximum likelihood (ML) algorithm is the most common and effective parameter estimation method. However, when dealing with small sample and low signal-to-noise ratio (SNR), threshold effects are resulted and estimation performance degrades greatly. It is proved that support vector machine (SVM) is suitable for small sample. Furthermore, it is verified that, by taking single-tone sinusoidal frequency estimation, for example, and integrating data analysis and experimental validation, if LS-SVR's parameters are set appropriately, not only the LS-SVR-based data preprocessing can ensure the single-tone sinusoid and AWGN channel characteristics of original signals well, but also the frequency estimation performance improves.

Development of various algorithms related to communication networks and sensor networks is a supplement to control and filtering schemes. The article "A heuristic algorithm for solving triangle packing problem" by R. Wang et al. studies the triangle packing problem by a new kind of method, which has broad application prospects in material processing, network resource optimization, and so forth. Such concepts as angle region, corner-occupying action, corner-occupying strategy, and edge-conjoining strategy are presented in this paper. In addition, an algorithm of edge conjoining and corner occupying is designed, which is to obtain an approximate solution. The paper entitled "Research on the architecture of a basic reconfigurable information communication network" by R. Wang et al. provides the theory system for the basic reconfigurable information communication network based on the analysis of present problems on the Internet and summarizes the root of these problems. It also provides an in-depth discussion about the related technologies and 
the prime components of the architecture. The problem of distributed estimation fusion over peer-to-peer asynchronous sensor networks with random packet dropouts is concerned in "Distributed asynchronous fusion algorithm for sensor networks with packet losses" by T. Chu et al. A distributed asynchronous fusion algorithm is proposed via the covariance intersection method. First, local estimator is developed in an optimal batch fashion by constructing augmented measurement equations. Then the fusion estimator is designed to fuse local estimates in the neighborhood. Both local estimator and fusion estimator are developed by taking into account the random packet losses. The presented estimation method improves local estimates and reduces the estimate disagreement. Aim at the problems and insufficiency of dynamic integrity measurement method, a dynamic integrity measurement model based on Memory Paging Mechanism, is proposed in the paper entitled "Research on dynamic integrity measurement model based on memory paging mechanism" by $\mathrm{C}$. Chang et al. The model takes memory pages of executable subjects as measurement objects. When the pages are scheduled into memory, the measurement points are inserted, the pages are measured, and their integrities are verified. The model is able to insure the integrity and trusty of each executable page and assure that the integrity of the whole executable subjects is not destroyed. To verify this model, XEN hypercall mechanism is used to acquire executable subjects' pages scheduled into memory, and the integrity measurement and verification codes are put into hypercall handler.

\section{Acknowledgments}

This special issue is a timely reflection of the recent research progress in the area of filtering and control for unreliable communication in the discrete-time domain. We would like to acknowledge all authors for their efforts in submitting high-quality papers. We are also very grateful to the reviewers for their thorough and on time reviews of the papers.

Guoliang Wei Lifeng Ma Zidong Wang 


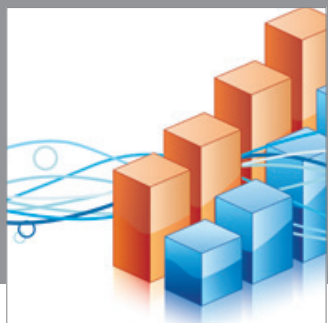

Advances in

Operations Research

mansans

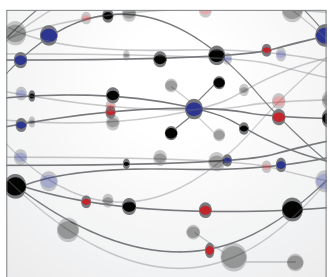

The Scientific World Journal
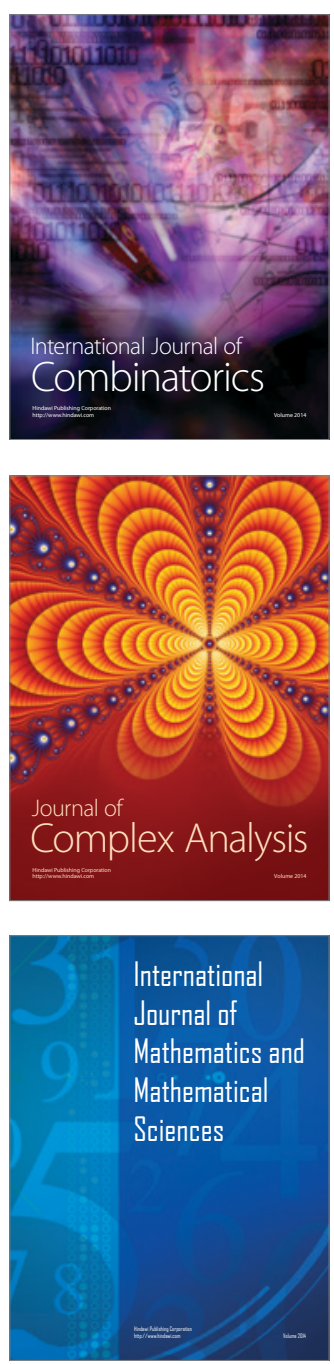
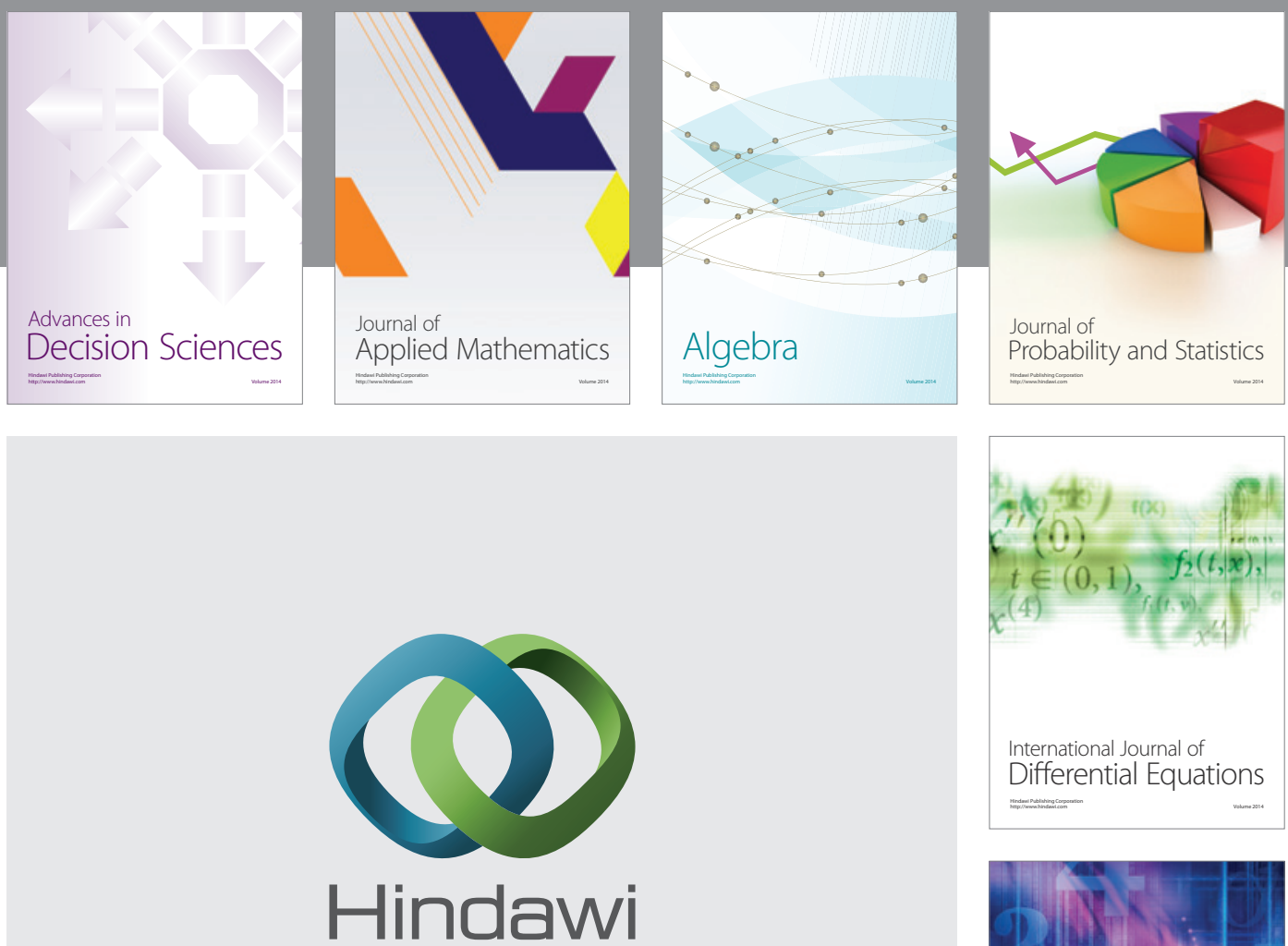

Submit your manuscripts at http://www.hindawi.com
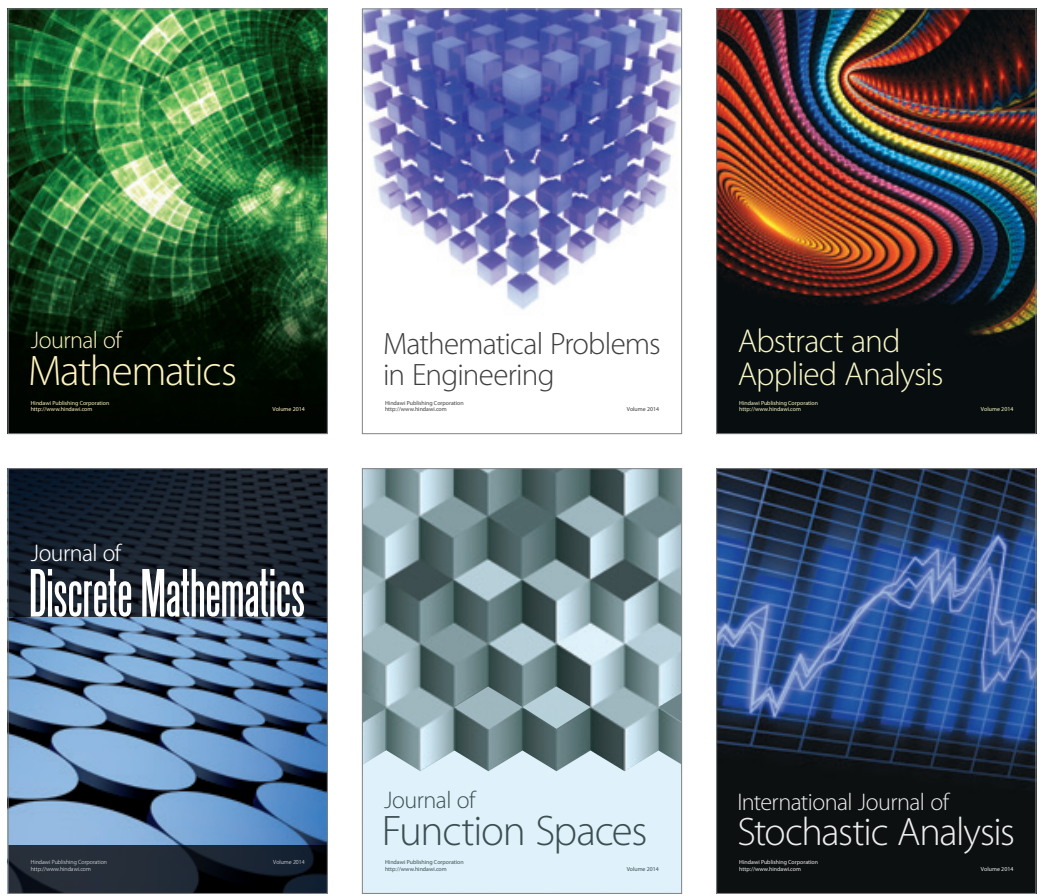

Journal of

Function Spaces

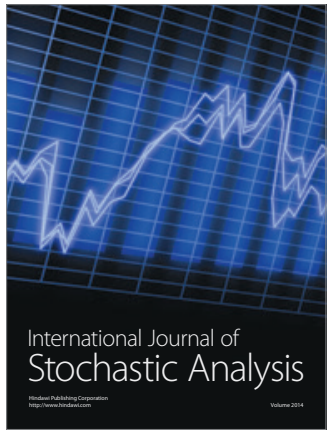

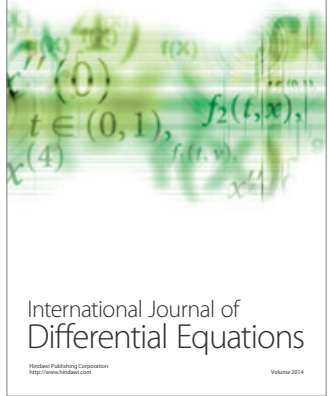
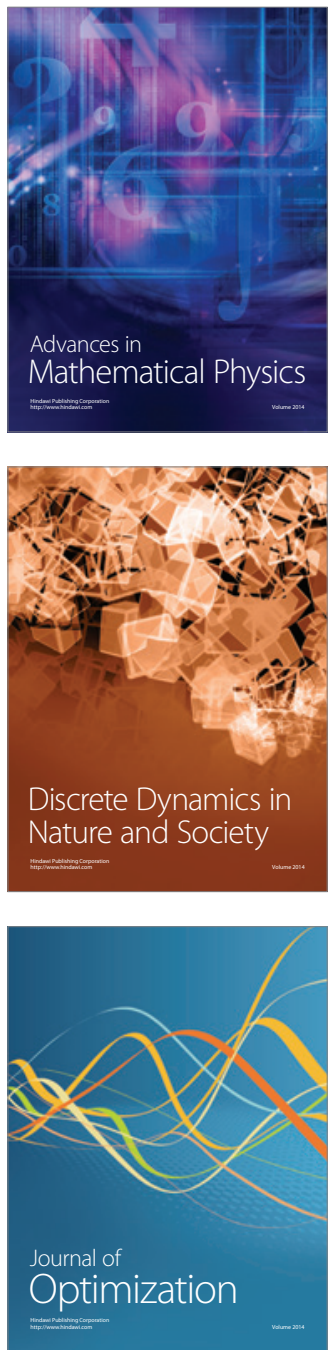